\title{
Anatomical and Functional Estimations of Brachial Artery Diameter and Elasticity Using Oscillometric Measurements with a Quantitative Approach
}

\author{
Keiichiro Yoshinaga a, b Satoshi Fujiic Yuuki Tomiyamab \\ Keisuke Takeuchi ${ }^{d}$ Nagara Tamaki ${ }^{b}$ \\ a Molecular Imaging Research Center, National Institute of Radiological Sciences, Chiba, \\ ${ }^{b}$ Department of Nuclear Medicine, Hokkaido University Graduate School of Medicine, \\ Sapporo, 'Department of Laboratory Medicine, Asahikawa Medical University, Asahikawa, \\ and ${ }^{\mathrm{d}}$ Faculty of Health Sciences, Hokkaido University Graduate School of Medicine, \\ Sapporo, Japan
}

\section{Key Words}

Atherosclerosis · Brachial artery · Elasticity · Oscillometric measurement · Ultrasound

\begin{abstract}
Noninvasive vascular function measurement plays an important role in detecting early stages of atherosclerosis and in evaluating therapeutic responses. In this regard, recently, new vascular function measurements have been developed. These new measurements have been used to evaluate vascular function in coronary arteries, large aortic arteries, or peripheral arteries. Increasing vascular diameter represents vascular remodeling related to atherosclerosis. Attenuated vascular elasticity may be a reliable marker for atherosclerotic risk assessment. However, previous measurements for vascular diameter and vascular elasticity have been complex, operator-dependent, or invasive. Therefore, simple and reliable approaches have been sought. We recently developed a new automated oscillometric method to measure the estimated area (eA) of a brachial artery and its volume elastic modulus $\left(\mathrm{V}_{\mathrm{E}}\right)$. In this review, we further report on this new measurement and other vascular measurements. We report on the reliability of the new automated oscillometric measurement of eA and $V_{\mathrm{E}}$. Based on our findings, this measurement technique should be a reliable approach, and this modality may have practical application to automatically assess muscular artery diameter and elasticity in clinical or epidemiological settings. In this review, we report the characteristics of our new oscillometric measurements and other related vascular function measurements.
\end{abstract}


Yoshinaga et al.: Anatomical and Functional Estimations of Brachial Artery Diameter and Elasticity Using Oscillometric Measurements with a Quantitative Approach

Table 1. Noninvasive vascular function measurements

\begin{tabular}{|c|c|c|c|c|}
\hline Test & Vascular bed & Definition & Normal value & Equation \\
\hline PWV & Aorta & $\begin{array}{l}\text { Speed of travel of the pulse along } \\
\text { an arterial segment }\end{array}$ & $\begin{array}{l}\text { cfPWV }<10 \mathrm{~m} / \mathrm{s} \\
\text { baPWV }<18 \mathrm{~m} / \mathrm{s}\end{array}$ & Distance $/ \Delta \mathrm{t}(\mathrm{cm} / \mathrm{s})$ \\
\hline Stiffness index & Carotid artery & $\begin{array}{l}\text { Ratio of logarithm (systolic/ } \\
\text { diastolic pressures) to (relative } \\
\text { change in diameter) }\end{array}$ & Under investigation & $\begin{array}{l}\beta=\ln (\mathrm{Ps} / \mathrm{Pd}) /[(\mathrm{Dd}-\mathrm{Ds}) / \\
\mathrm{Dd}] \text { (nondimensional) }\end{array}$ \\
\hline $\mathrm{V}_{\mathrm{E}}$ & Brachial artery & $\begin{array}{l}\text { Pressure step required for } \\
\text { (theoretical) } 100 \% \text { increase in } \\
\text { volume }\end{array}$ & Under investigation & $\Delta \mathrm{P} /(\Delta \mathrm{V} / \mathrm{V})(\mathrm{mm} \mathrm{Hg})$ \\
\hline FMD & Brachial arteries & $\begin{array}{l}\text { Vasodilatation response to } \\
\text { hyperemia }\end{array}$ & $\begin{array}{l}5-10 \% \text { (upper arm } \\
\text { occlusion) }\end{array}$ & $\begin{array}{l}\text { Arterial diameter at } \\
\text { hyperemia/arterial } \\
\text { diameter at rest }\end{array}$ \\
\hline Biomarker & Nonspecific & $\begin{array}{l}\text { Various vascular injury markers } \\
\text { and inflammation markers }\end{array}$ & - & - \\
\hline PET & $\begin{array}{l}\text { Coronary } \\
\text { resistance vessels }\end{array}$ & $\begin{array}{l}\text { Cold pressor test } \\
\text { No mediated vascular dilatation }\end{array}$ & $\begin{array}{l}30-60 \% \text { increase in } \\
\text { blood flow }\end{array}$ & $\begin{array}{l}\text { MBF during cold pressor } \\
\text { stress/MBF at rest }\end{array}$ \\
\hline
\end{tabular}

cfPWV = Carotid-femoral PWV; $\mathrm{P}=$ pressure; $\mathrm{D}=$ diameter; $\mathrm{V}=$ volume; $\mathrm{MBF}=$ myocardial blood flow.

\section{Introduction}

Noninvasive vascular function measurements have been used to detect early stages of atherosclerosis and have contributed to the risk assessment of subjects with risk factors for coronary artery disease [1-3]. Vascular functional measurements include vascular anatomical changes, arterial stiffness, blood flow changes, and vascular elasticity in several vascular beds from microcirculation in coronary arteries and brachial arteries to that in large arteries and the aorta. Several diagnostic modalities have been developed to noninvasively measure vascular function. Among these, ultrasound is widely used in clinical and community study settings [4]. Ultrasound can estimate vascular anatomical changes and functional changes under appropriate stress protocols (table 1) [2]. Upper-arm ultrasound measurements can be used to estimate vascular diameter, endothelial function using flow-mediated dilatation (FMD), and endothelial-independent vasodilator function using nitroglycerin (NTG) stress [5, 6]. In this regard, ultrasound measurement plays an important role in estimating the function of peripheral arteries and is considered to be one of the standard vascular function measurements. However, data acquisition and data analysis require training, and there can be an issue with the reproducibility of ultrasound measurements [7]. Therefore, many investigators have developed alternative new approaches for vascular function measurements to evaluate anatomical changes or functional abnormalities. We have developed positron emission tomography (PET) to measure coronary endothelial function $[1,8,9]$. PET is accurate and coronary specific, but it can be performed in a limited number of facilities and is used mainly as a research tool. For wider clinical use, several noninvasive measurements have also been developed, including pulse wave velocity (PWV) to measure arterial stiffness in the large arteries [10-13]. The original PWV method of carotid-femoral PWV is the reference standard and can be used to evaluate large artery stiffness [13]. Another approach involving brachialankle PWV (baPWV) has been developed. baPWV can also be used to easily measure PWV 
with blood pressure (BP) measurement cuffs and has been validated against invasively derived aortic PWV [14]. Therefore, baPWV is widely applied in clinical settings and is useful in cardiovascular outcome predictions in the Asian population $[10,15,16]$. However, PWV is an index of large artery stiffness associated with structure $[3,17]$. In addition, PWV is influenced by BP and heart rate, because elevated BP increases the blood wall tension and reduces vascular elasticity $[18,19]$. Increased wall tension due to elevated BP increases the velocity of the bloodstream and hence increases the PWV. The influence of BP may be one of the major limitations of PWV. Cardio-ankle-vascular index (CAVI) is not affected by BP. Data acquisition with CAVI is basically similar to that with PWV but as with PWV, CAVI evaluates the vascular function of only large arteries, a fact that represents a major limitation of this approach [20]. Augmentation index is also influenced by BP and heart rate. Therefore, alternative noninvasive approaches that are not influenced by BP or heart rate are also being sought. In contrast, vascular elasticity is not considered to be influenced by a patient's BP at the time of measurement and can be measured through an oscillometric approach [11,21]. We therefore aimed to make new vascular anatomical and functional measurements using an oscillometric approach [22].

\section{Concept of Oscillometric Approach for Evaluating Vascular Elasticity}

Arterial stiffness can be measured using the information regarding arterial pressure and diameter changes. Vascular elastic modulus $\left(\mathrm{V}_{\mathrm{E}}\right)$ is the pressure change required for $100 \%$ increase in vascular diameter or pressure change per square centimeter for $100 \%$ extension [21]. $\mathrm{V}_{\mathrm{E}}$ is defined as the change in the BP-CP (cuff pressure) difference (transmural pressure) per $1 \%$ increase in the cross-sectional area at P1, where P1 = P0 + $40 \mathrm{~mm} \mathrm{Hg} \mathrm{[22].} \mathrm{The} \mathrm{highest}$ $\mathrm{CP}$ can cause complete collapse of the brachial artery. The highest CP may be slightly greater than BP. Therefore, P0 at diastole on the transmural pressure axis was set at less than zero and is associated with complete vascular occlusion [22]. Therefore, vascular elasticity is only minimally influenced by BP at the time of measurement and can be measured through an oscillometric approach $[11,21]$. If BP increases, the arterial wall will become less distensible. In this regard, vascular elasticity will increase in parallel with the increasing BP. To minimize this BP influence, pressure would be applied to the outside of the artery using a BP cuff. With the appropriate $\mathrm{CP}$, the transmural pressure can be maintained. Akimoto et al. [23] addressed this concept in the discussion section of their article. We applied this concept to our measurements. Our system estimated $\mathrm{V}_{\mathrm{E}}$ at the same intramural pressure point as $\mathrm{P} 1$. In response to rising $\mathrm{BP}$, our system raised $\mathrm{CP}$, thereby maintaining constant transmural pressure on the vascular wall. Therefore, a patient's BP at the time of measurement may not have much impact on vascular elasticity. As shown in figure 5, our system initially measures BP. As the second step, our system determines the required $\mathrm{CP}$ to induce complete arterial occlusion at diastole. This pressure is defined as P0. Theoretically speaking, using constant or equal intramural pressure can minimize the influence of BP. However, this theory was developed through physics laboratory observation, and human arteries do not behave in the same way as tube models. Therefore, our system can only minimize the influence of BP. Although our system does not provide an oscillogram report, our system automatically evaluated the oscillogram. When the envelope is free from error, our system proceeds with further measurements. We took measurements at 5 points to obtain the pressure-volume curve (fig. 5). Increasing the number of measurement points may improve the accuracy of measurements. However, increasing the number of sample points requires longer data acquisition time, and therefore, we have maintained 5 measurement points. Ideally, the number of points of measurement should be increased, and doing so should be a next step. 
Yoshinaga et al.: Anatomical and Functional Estimations of Brachial Artery Diameter and Elasticity Using Oscillometric Measurements with a Quantitative Approach

Fig. 1. Overview of automated oscillometric measurements. The measurement was performed with the patient in supine position, and triple lumen cuffs were put on the upper arm.

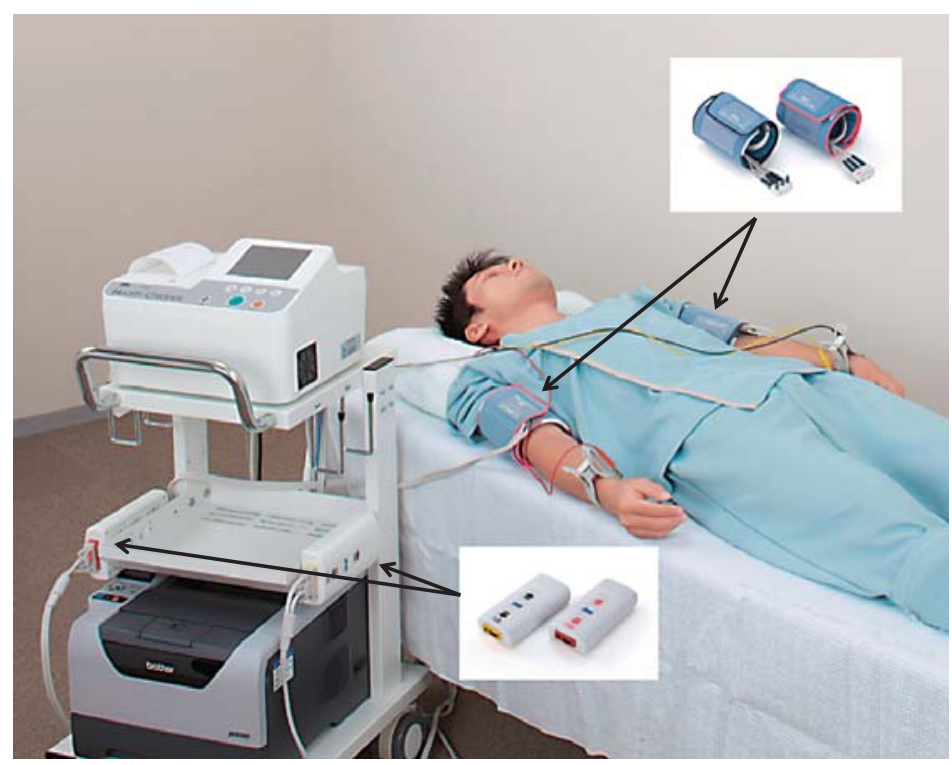

Previous studies have estimated the $V_{E}$ using different approaches. Bank et al. [11] estimated $V_{E}$ using a water-filled BP cuff with an external ultrasound. Kinlay et al. [24] estimated this marker using intravascular ultrasound and a catheter. These approaches measured vascular area changes during various pressure changes. These approaches obtained BP through arterial catheter or applanation tonometry, approaches that were either complex or invasive and therefore not suitable for clinical practice. For these reasons, we aimed to develop new vascular anatomical and functional measurements using an oscillometric approach [22].

The system we developed can automatically evaluate the estimated area $(\mathrm{eA})$ and $\mathrm{V}_{\mathrm{E}}$. The newly developed device is the Health Chronos TM-2771 prototype (A\&D Company, Tokyo, Japan; fig. 1,2) [22, 25]. This system estimates the absolute value of cross-sectional vascular area instead of vascular volume. Thus, the original $V_{E}$ equation was modified as follows [25]: $\left[\mathrm{V}_{\mathrm{E}}=\Delta\right.$ pressure $/(100 \times \Delta$ area/area $\left.) \mathrm{mm} \mathrm{Hg} / \%\right]$. The principal concept of $\mathrm{V}_{\mathrm{E}}$ estimation was to evaluate the association between $\Delta$ area and $\Delta$ pressure, while the blood vessel remained circular. The correct measurements should be performed with CP below a certain point so that the vessel remains circular and does not buckle. The main control unit creates various fixed precise volumes of air, which are capable of changing the tube size, giving rise to the so-called tube law [26]. Differences between intramural BP and CP can be obtained using oscillometric measurements. This is the basic concept of this automated system. Using the pressure-volume curves, this oscillometric method quantitatively estimates vascular eA and $\mathrm{V}_{\mathrm{E}}$ (fig. 3).

As mentioned earlier, $\mathrm{V}_{\mathrm{E}}$ is theoretically not influenced by BP. In fact, our data showed no correlations between the systolic BP $(r=0.19, p=0.47)$ or diastolic BP $(r=0.19, p=0.48)$ and $V_{E}$ (fig. $\left.4 a, b\right)$. When vascular function measurements for risk assessments are applied, any index influenced by BP may have an impact on the data. Based on the current data, being able to use $V_{\mathrm{E}}$ to determine vascular function measurements is an important feature of the current measurement approach. However, the sample size of this analysis was small. Therefore, we definitely need further study to confirm this finding using a larger study population. 
Yoshinaga et al.: Anatomical and Functional Estimations of Brachial Artery Diamete and Elasticity Using Oscillometric Measurements with a Quantitative Approach

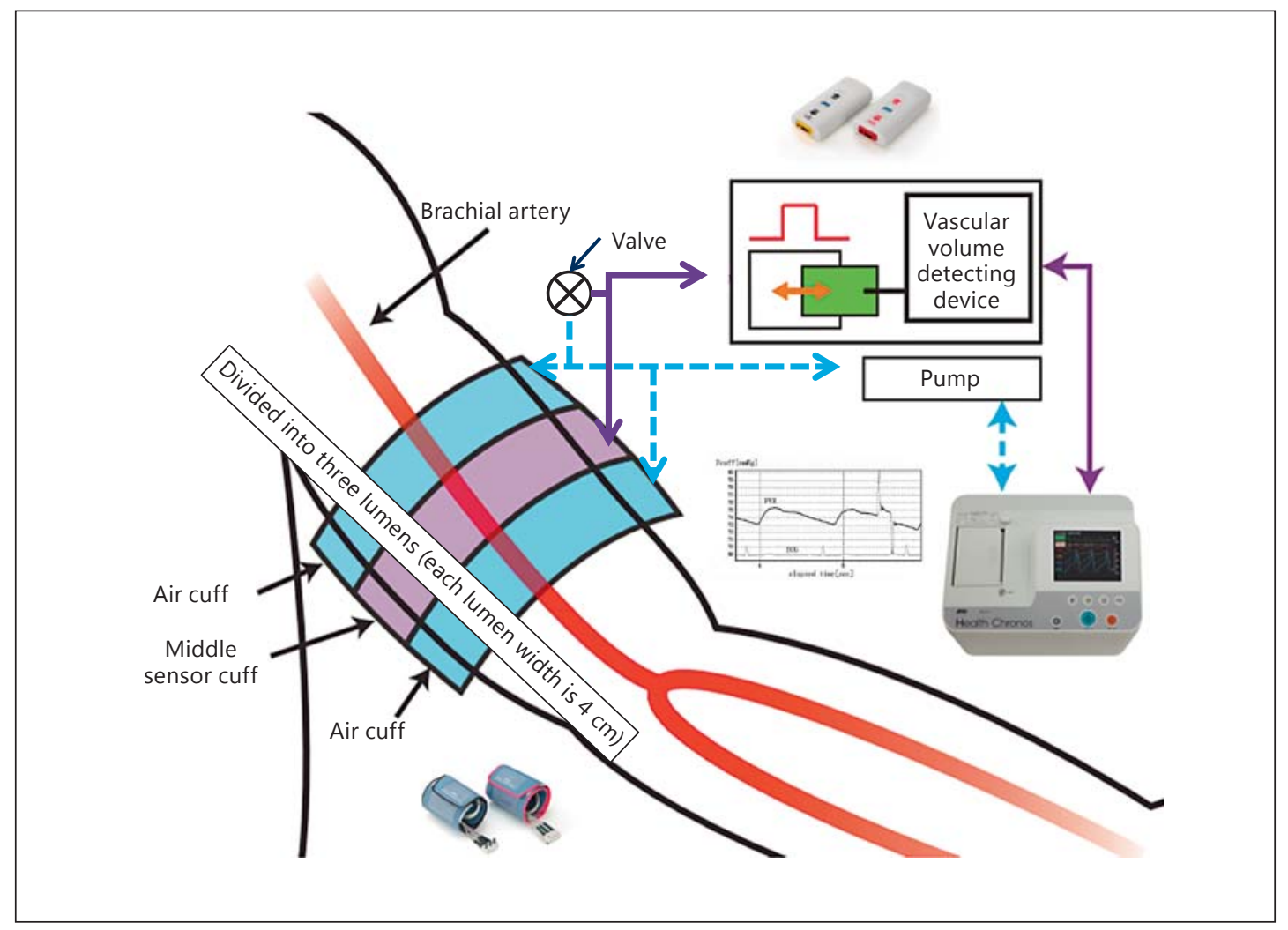

Fig. 2. Oscillometric measurement setup. The triple lumen cuff had a central pressure sensor cuff and two outside cuffs. The central part of the triple lumen cuff detected the exact vascular volume change. A main control unit generated a precise amount of air within all 3 cuffs. After the precise amount of air was inserted, the two tubes to the outside cuffs were occluded. These amounts of air changed the vascular volume and exerted a steady pressure on the vessel. Once the outer cuffs were inflated, the sensor on the central cuff was activated through the main control unit to detect changes in vascular volume (as noted in Tomiyama et al. [22]; the authors hold copyright). The central pressure sensor cuff detected BP-CP differences during the heartbeat cycle. Using this information, the main control unit created pressure-volume curves.

\section{Concept of Oscillometric Approach to Evaluate Vascular Diameter}

Vascular dysfunction appears prior to changes in morphology [27, 28]. Therefore, our group has focused on developing functional imaging to detect the earlier stages of atherosclerosis [1,9]. On the other hand, Otsuka and Munakata [29] pointed out the importance of vascular diameter changes resulting from vascular remodeling. They noted that another advantage of this oscillometric measurement is the ability to estimate the cross-sectional vascular area (eA) [22, 29].

To estimate the eA, the brachial arterial volume was divided by the length of the central cuff. The eA was estimated using pressure-volume curves (fig. 3). The eA was estimated when $\mathrm{BP}$ minus $\mathrm{CP}$ was identical to diastolic BP. Thus, $\mathrm{CP}$ should be $0 \mathrm{~mm} \mathrm{Hg}$. At this point, the blood vessel should be dilated and should reflect the exact cross-sectional vascular area. In fact, the eA was closely correlated with the arterial diameter evaluated by ultrasound measurements [22]. Unlike Kinlay's approach [24], our approach cannot visualize vascular morphological change, but it can at least evaluate important vascular diameter or cross-sectional area information. The latter point presents an additional advantage of this approach over other oscil- 
Yoshinaga et al.: Anatomical and Functional Estimations of Brachial Artery Diameter and Elasticity Using Oscillometric Measurements with a Quantitative Approach

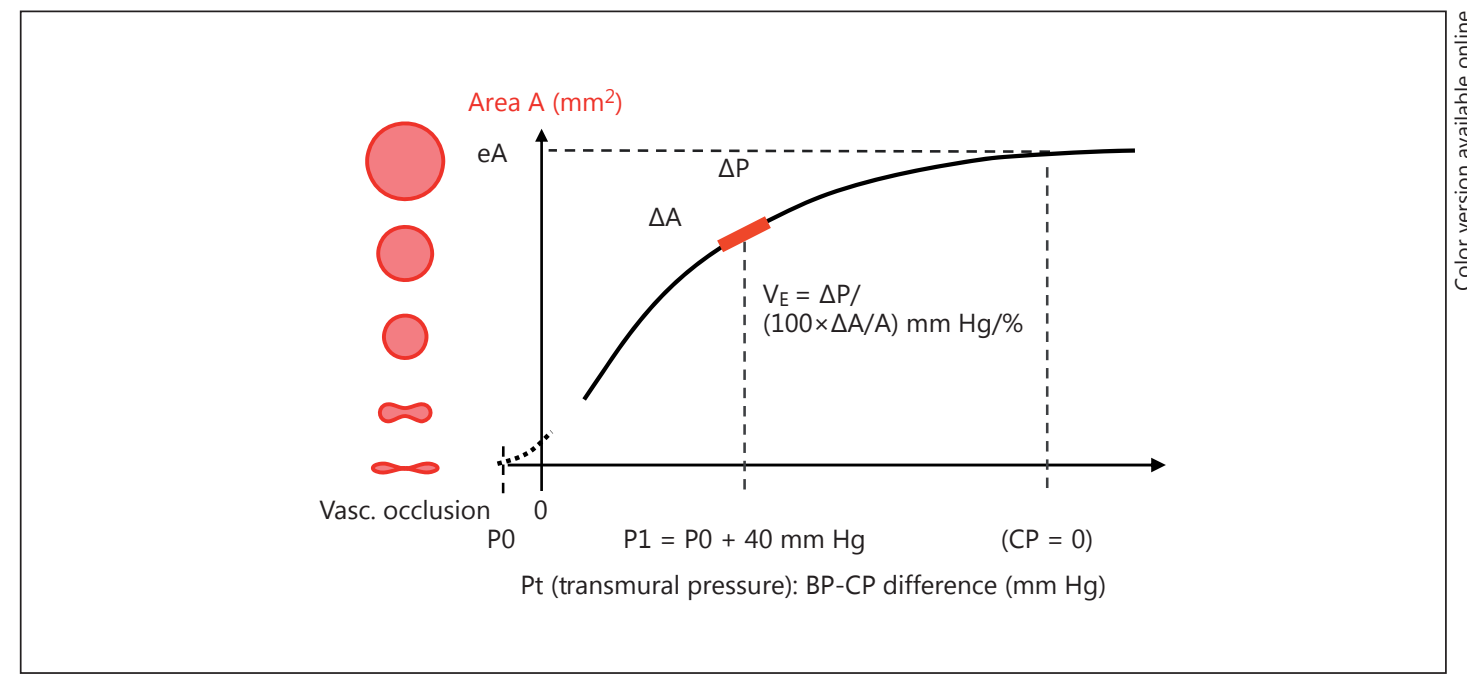

Fig. 3. Estimated cross-sectional area $(\mathrm{eA})$ and volume elastic modulus $\left(\mathrm{V}_{\mathrm{E}}\right)$ obtained using pressure-volume curves. In the case that $\mathrm{CP}$ becomes zero, the calculated cross-sectional vascular area should become the baseline vascular area. P0 is the point of transmural pressure at which the brachial artery is completely occluded. $\mathrm{P} 1$ is the $\mathrm{V}_{\mathrm{E}}$ measured at the point of transmural pressure. After estimating P0, P1 can be determined using the following equation. $\mathrm{P} 1-\mathrm{P} 0=40 \mathrm{~mm} \mathrm{Hg}$. $\Delta \mathrm{A}$ is the difference in vascular area. $\Delta \mathrm{P}$ is the difference in transmural pressure.

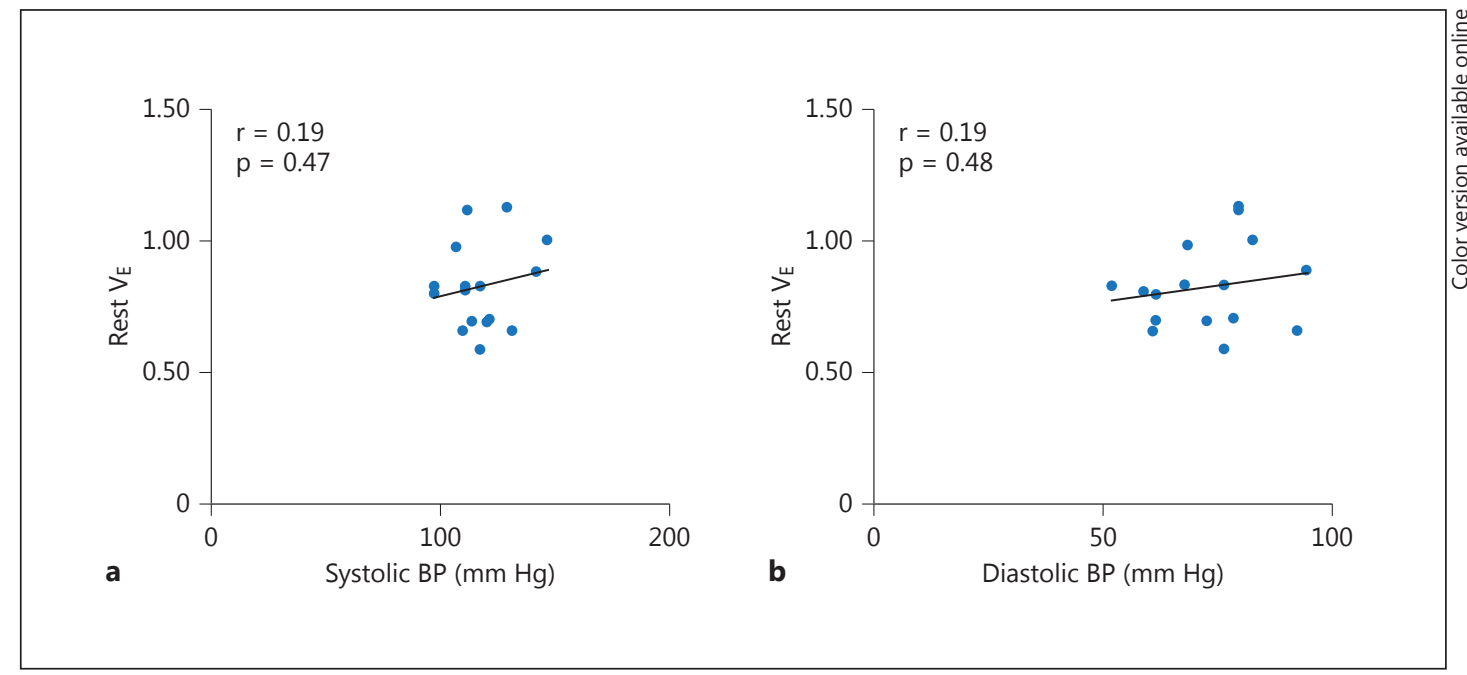

Fig. 4. Correlation between $\mathrm{BP}$ and $\mathrm{V}_{\mathrm{E}}$. a Correlation between systolic $\mathrm{BP}$ and $\mathrm{V}_{\mathrm{E}}$. $\mathbf{b}$ Correlation between diastolic BP and $\mathrm{V}_{\mathrm{E}}$.

lometric approaches to vascular function measurements including PWV and CAVI. Unlike upper-arm ultrasound, our system does not directly measure the brachial artery's eA and $V_{\mathrm{E}}$. These measurements come mainly from the brachial artery. However, there are some other small arteries in the upper arm. Therefore, our system evaluates the sum of arterial structural and functional information. The aggregate nature of this information represents a limitation and a disadvantage of this technique compared to upper-arm ultrasound. 
Yoshinaga et al.: Anatomical and Functional Estimations of Brachial Artery Diamete and Elasticity Using Oscillometric Measurements with a Quantitative Approach

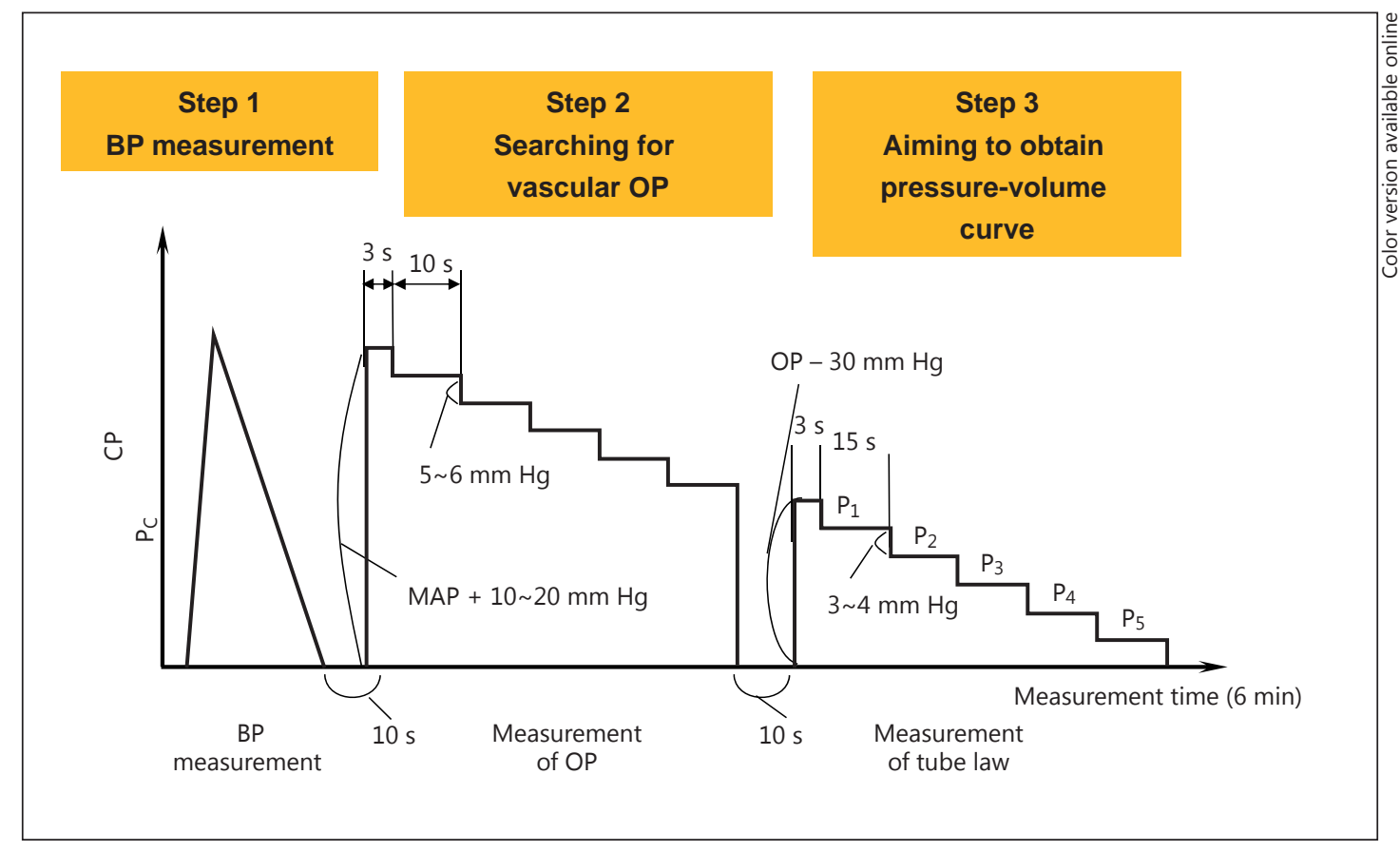

Fig. 5. Detail of measurement protocol with $\mathrm{CP}$ protocol. $\mathrm{MAP}=$ Mean arterial pressure; $\mathrm{OP}=$ occlusion pressure.

\section{Reliability of Oscillometric Measurements}

Any new diagnostic test requires validation before it can be used in clinical settings. To be validated, a new approach must have the same accuracy as that of established methods [8, 14], and its test-retest reproducibility must be evaluated [30]. In the previous study, we showed a high correlation between eA measurements and ultrasound measurements [22]. In brief, we performed the validity assessments and reproducibility assessment of eA and $V_{E}$ in 16 normal individuals (age $35.2 \pm 13.1$ years). These normal volunteers underwent oscillometric measurements and brachial ultrasound at rest. The interval between the ultrasound and oscillometric measurement was $4.9 \pm 3.7$ days. The rest eA correlated with ultrasoundmeasured brachial artery area $(\mathrm{r}=0.77, \mathrm{p}<0.001)$. The oscillometric approach to the eA was thus validated through the standard approach. Oscillometric measurement was performed twice on different days with the mean interval between the two oscillometric measurements being $7.2 \pm 5.2$ days. Rest eA and rest $\mathrm{V}_{\mathrm{E}}$ showed good reliability [eA: intraclass correlation coefficient $($ ICC $)=0.88, \mathrm{~V}_{\mathrm{E}}$ : ICC $\left.=0.78\right]$. Neither index showed differences between the first and second measurements. The eA was significantly correlated with ultrasound measurements and showed a high ICC, indicating a high level of reliability of measurements. The reliability of eA was slightly higher than that of $V_{E}$. The slightly lower reproducibility of $V_{E}$ may be due to differences in individual conditions under which the two measurements were performed on different days. It is widely recognized that vascular function is influenced by several factors [7]. The current approach is automated and is a reliable technique from a technical point of view, even though $V_{\mathrm{E}}$ measurement requires careful subject preparation and careful data acquisition conditions similar to those for ultrasound study [7].

We further evaluated the reliability of oscillometric measurements by applying NTG stress. NTG induces maximal vasodilatation [7]. Unlike FMD or other pharmacological stress, 
Yoshinaga et al.: Anatomical and Functional Estimations of Brachial Artery Diamete and Elasticity Using Oscillometric Measurements with a Quantitative Approach

NTG is not influenced by a subject's condition and can almost always induce maximal vasodilatation [31]. Therefore, NTG stress may be a useful stress technique for evaluating the reliability of new vascular functional tests. In our previous study, the eA significantly increased after NTG stress, which was similar to what ultrasound measurements showed. The NTG stress study further confirmed the reliability of oscillometric measurements. Based on our findings, our previous study suggests the importance of stress studies in evaluating new methods of measuring vascular function.

\section{Practical Aspects of Oscillometric Measurements}

Noninvasive vascular functional measurements are usually used for epidemiological research studies and risk stratifications in patients who have atherosclerotic risk factors. In this regard, simple data acquisition and shorter data acquisition times are desirable. For this purpose, PWV can be performed within a few minutes, similar to the case with ankle-brachial index measurements, and is suitable for larger epidemiological studies [15]. In contrast, FMD usually takes 30-40 min [5, 7]. To generate the pressure-volume curves, the main control unit of our oscillometric approach creates several different pressures within the BP cuff following initial BP measurements (fig. 5). Each cuff occlusion takes $10 \mathrm{~s}$, and measurement of occlusion pressure has 5 steps. After this measurement, additional measurement for the tube law is performed. Therefore, the total measurement time is $6 \mathrm{~min}$. This system requires a relatively short data acquisition time and can therefore be applied for many patients in clinical settings. In addition, when the measurements are taken, the BP cuff needs to be put on the upper arm. This procedure is almost identical to taking a standard BP measurement and does not require specific skills. Among the noninvasive vascular function measurements, this oscillometric measurement should be categorized as a simple automated approach.

\section{Future Directions for Oscillometric Measurements}

The goal of developing any new method of measuring vascular function is to achieve wide clinical use. In this regard, FMD, PWV, and CAVI have been useful in showing arteriosclerosis risk stratifications in various study populations [4, 32,33]. As the initial step, we evaluated the age-related vascular diameter change and elasticity change. Vascular diameter increases and vascular function alters in association with aging [34-37]. According to our previous study, eA and $V_{\mathrm{E}}$ were correlated with increasing age $\left(\mathrm{eA}: \mathrm{r}=0.54, \mathrm{p}=0.045 ; \mathrm{V}_{\mathrm{E}}: \mathrm{r}=0.81, \mathrm{p}<\right.$ $0.001)$ [22]. These data imply that these oscillometric measurements can be used in clinical settings. The $V_{\mathrm{E}}$ is not influenced by a patient's BP at the time of measurement. Many treatments to reduce atherosclerosis may result in a change to BP. Parameters not influenced by $\mathrm{BP}$ can be examined to reliably evaluate the therapeutic effects of hypertension treatments on vascular function, and therefore, our new system could play an important role in evaluating the effects of treatments. This possibility is currently under investigation.

The exact mechanism of attenuated $V_{\mathrm{E}}$ is also important, and our previous study did not evaluate the exact mechanisms. Using BP cuff occlusion is somewhat similar to performing FMD. In this regard, attenuated $V_{E}$ may partially reflect endothelial dysfunction. However, we have not confirmed this possibility, and this possibility is still open to examination. 
Yoshinaga et al.: Anatomical and Functional Estimations of Brachial Artery Diamete and Elasticity Using Oscillometric Measurements with a Quantitative Approach

\section{Conclusion}

We have developed the new quantitative automated oscillometric measurement technique to assess brachial artery cross-sectional area and vascular elasticity. This measurement technique can detect morphological and functional changes simultaneously. Therefore, this modality may have practical application in quantitatively assessing muscular artery elasticity and diameter responses in both clinical settings and epidemiological studies. These possibilities are currently under investigation.

\section{Acknowledgments}

This study was supported in part by grants from the Japanese Ministry of Education, Culture, Sports, Science and Technology (HOUGA Grant No. 24659550), grants from the Innovation Program of the Japan Science and Technology Agency, and the Smoking Research Foundation (2011-2015). Y.T. is supported by the Research Assistant Program, Hokkaido University Graduate School of Medicine. K.Y. is supported by the Imura Clinical Research Award (Adult Vascular Disease Research Foundation). The manuscript has been reviewed by a North American English-language professional editor, Ms. Holly Beanlands. The authors also thank Ms. Holly Beanlands for critical reading of the manuscript.

\section{Disclosure Statement}

This work was in part supported by A\&D Company, Limited (Tokyo, Japan).

\section{References}

1 Yoshinaga K, Manabe 0, Tamaki N: Assessment of coronary endothelial function using PET. J Nucl Cardiol 2011;18:486-500.

2 Flammer AJ, Anderson T, Celermajer DS, Creager MA, Deanfield J, Ganz P, et al: The assessment of endothelial function: from research into clinical practice. Circulation 2012;126:753-767.

3 Blacher J, Safar ME: Large-artery stiffness, hypertension and cardiovascular risk in older patients. Nat Clin Pract Cardiovasc Med 2005;2:450-455.

4 Kallio K, Jokinen E, Raitakari OT, Hamalainen M, Siltala M, Volanen I, et al: Tobacco smoke exposure is associated with attenuated endothelial function in 11-year-old healthy children. Circulation 2007;115:32053212.

5 Celermajer DS, Adams MR, Clarkson P, Robinson J, McCredie R, Donald A, et al: Passive smoking and impaired endothelium-dependent arterial dilatation in healthy young adults. N Engl J Med 1996;334:150-154.

6 Ochi N, Yoshinaga K, Ito YM, Tomiyama Y, Inoue M, Nishida M, et al: Comprehensive assessment of impaired peripheral and coronary artery endothelial functions in smokers using brachial artery ultrasound and oxygen15-labeled water PET. J Cardiol 2015, DOI: 10.1016/j.jjcc.2015.10.006.

7 Corretti MC, Anderson TJ, Benjamin EJ, Celermajer D, Charbonneau F, Creager MA, et al: Guidelines for the ultrasound assessment of endothelial-dependent flow-mediated vasodilation of the brachial artery: a report of the International Brachial Artery Reactivity Task Force. J Am Coll Cardiol 2002;39:257-265.

8 Yoshinaga K, Manabe O, Katoh C, Chen L, Klein R, Naya M, et al: Quantitative analysis of coronary endothelial function with generator-produced 82Rb PET: comparison with 150-labelled water PET. Eur J Nucl Med Mol Imaging 2010;37:2233-2241.

9 Yoshinaga K, Tomiyama Y, Suzuki E, Tamaki N: Myocardial blood flow quantification using positron-emission tomography: analysis and practice in the clinical setting. Circ J 2013;77:1662-1671.

10 Townsend RR, Wilkinson IB, Schiffrin EL, Avolio AP, Chirinos JA, Cockcroft JR, et al: Recommendations for improving and standardizing vascular research on arterial stiffness: a scientific statement from the American Heart Association. Hypertension 2015;66:698-722.

11 Bank AJ, Wang H, Holte JE, Mullen K, Shammas R, Kubo SH: Contribution of collagen, elastin, and smooth muscle to in vivo human brachial artery wall stress and elastic modulus. Circulation 1996;94:3263-3270.

12 Usui Y, Takata Y, Inoue Y, Tomiyama H, Kurohane S, Hashimura Y, et al: Severe obstructive sleep apnea impairs left ventricular diastolic function in non-obese men. Sleep Med 2013;14:155-159. 
Yoshinaga et al.: Anatomical and Functional Estimations of Brachial Artery Diameter and Elasticity Using Oscillometric Measurements with a Quantitative Approach

13 Van Bortel LM, Laurent S, Boutouyrie P, Chowienczyk P, Cruickshank JK, De Backer T, et al: Expert consensus document on the measurement of aortic stiffness in daily practice using carotid-femoral pulse wave velocity. J Hypertens 2012;30:445-448.

14 Yamashina A, Tomiyama H, Takeda K, Tsuda H, Arai T, Hirose K, et al: Validity, reproducibility, and clinical significance of noninvasive brachial-ankle pulse wave velocity measurement. Hypertens Res 2002;25:359364.

15 Yamashina A, Tomiyama H, Arai T, Koji Y, Yambe M, Motobe H, et al: Nomogram of the relation of brachialankle pulse wave velocity with blood pressure. Hypertens Res 2003;26:801-806.

16 Vlachopoulos C, Aznaouridis K, Terentes-Printzios D, Ioakeimidis N, Stefanadis C: Prediction of cardiovascular events and all-cause mortality with brachial-ankle elasticity index: a systematic review and meta-analysis. Hypertension 2012;60:556-562.

17 Avolio A: Arterial stiffness. Pulse (Basel) 2013;1:14-28.

18 Giannattasio C, Vincenti A, Failla M, Capra A, Ciro A, De Ceglia S, et al: Effects of heart rate changes on arterial distensibility in humans. Hypertension 2003;42:253-256.

19 Haesler E, Lyon X, Pruvot E, Kappenberger L, Hayoz D: Confounding effects of heart rate on pulse wave velocity in paced patients with a low degree of atherosclerosis. J Hypertens 2004;22:1317-1322.

20 Shirai K, Utino J, Otsuka K, Takata M: A novel blood pressure-independent arterial wall stiffness parameter; cardio-ankle vascular index (CAVI). J Atheroscler Thromb 2006;13:101-107.

21 O’Rourke MF, Staessen JA, Vlachopoulos C, Duprez D, Plante GE: Clinical applications of arterial stiffness; definitions and reference values. Am J Hypertens 2002;15:426-444.

22 Tomiyama Y, Yoshinaga K, Fujii S, Ochi N, Inoue M, Nishida M, et al: Accurate quantitative measurements of brachial artery cross-sectional vascular area and vascular volume elastic modulus using automated oscillometric measurements: comparison with brachial artery ultrasound. Hypertens Res 2015;38:478-484.

23 Akimoto E, Kobayashi H, Kawaharada J, Shimazu H, Ito H, Kinoshita H: Noninvasive measurement of volume elastic modulus in human finger arteries during and after the LI4 and LI10 acupuncture. J Jpn Society Acupuncture and Moxibustion 1989;39:306-312.

24 Kinlay S, Creager MA, Fukumoto M, Hikita H, Fang JC, Selwyn AP, et al: Endothelium-derived nitric oxide regulates arterial elasticity in human arteries in vivo. Hypertension 2001;38:1049-1053.

25 Otsuka T, Munakata R, Kato K, Kodani E, Ibuki C, Kusama Y, et al: Oscillometric measurement of brachial artery cross-sectional area and its relationship with cardiovascular risk factors and arterial stiffness in a middle-aged male population. Hypertens Res 2013;36:910-915.

26 Whittaker RJ HM, Jensen OE, Waters SL: A rational derivation of a tube law from shell theory. Q J Mech Appl Math 2010;63:465-496.

27 Halcox JP, Donald AE, Ellins E, Witte DR, Shipley MJ, Brunner EJ, et al: Endothelial function predicts progression of carotid intima-media thickness. Circulation 2009;119:1005-1012.

28 Yoshinaga K, Katoh C, Noriyasu K, Iwado Y, Furuyama H, Ito Y, et al: Reduction of coronary flow reserve in areas with and without ischemia on stress perfusion imaging in patients with coronary artery disease: a study using oxygen 15-labeled water PET. J Nucl Cardiol 2003;10:275-283.

29 Otsuka T, Munakata R: Enlargement of the brachial artery: significance of measurement. Hypertens Res 2015; 38:459-460.

30 Manabe O, Yoshinaga K, Katoh C, Naya M, deKemp RA, Tamaki N: Repeatability of rest and hyperemic myocardial blood flow measurements with 82Rb dynamic PET. J Nucl Med 2009;50:68-71.

31 Meirelles Cde M, Leite SP, Montenegro CA, Gomes PS: Reliability of brachial artery flow-mediated dilatation measurement using ultrasound. Arq Bras Cardiol 2007;89:160-167, 176-183.

32 Mitchell GF, Hwang SJ, Vasan RS, Larson MG, Pencina MJ, Hamburg NM, et al: Arterial stiffness and cardiovascular events: the Framingham Heart Study. Circulation 2010;121:505-511.

33 Shirai K, Hiruta N, Song M, Kurosu T, Suzuki J, Tomaru T, et al: Cardio-ankle vascular index (CAVI) as a novel indicator of arterial stiffness: theory, evidence and perspectives. J Atheroscler Thromb 2011;18:924-938.

34 Green DJ, Swart A, Exterkate A, Naylor LH, Black MA, Cable NT, et al: Impact of age, sex and exercise on brachial and popliteal artery remodelling in humans. Atherosclerosis 2010;210:525-530.

35 Hu H, Cui H, Han W, Ye L, Qiu W, Yang H, et al: A cutoff point for arterial stiffness using the cardio-ankle vascular index based on carotid arteriosclerosis. Hypertens Res 2013;36:334-341.

36 Glagov S, Weisenberg E, Zarins CK, Stankunavicius R, Kolettis GJ: Compensatory enlargement of human atherosclerotic coronary arteries. N Engl J Med 1987;316:1371-1375.

37 Safar ME, Levy BI, Struijker-Boudier H: Current perspectives on arterial stiffness and pulse pressure in hypertension and cardiovascular diseases. Circulation 2003;107:2864-2869. 